\title{
An approximate theory for substructure propagation in clusters
}

\author{
D. A. Prokhorov ${ }^{1,2}$ and F. Durret ${ }^{1}$ \\ 1 Institut d'Astrophysique de Paris, CNRS, UMR 7095, Université Pierre et Marie Curie, 98bis Bd Arago, 75014 Paris, France \\ e-mail: prokhoro@iap.fr \\ 2 Moscow Institute of Physics and Technology, Institutskii lane, 141700 Moscow Region, Dolgoprudnii, Russia
}

Received 26 March 2007 / Accepted 2 August 2007

\section{ABSTRACT}

\begin{abstract}
Aims. The existence of dark matter can be proved in an astrophysical context by the discovery of a system in which the observed baryons and the inferred dark matter are spatially segregated, such as the bullet cluster (1E0657-558). The full descriptions of the dark matter halo and X-ray gas substructure motions are necessary to forecast the location of the dark halo from X-ray maps, which can be confirmed by the detection of a galaxy concentration or by gravitational lensing.

Methods. We present an analytical hydrodynamic model to determine the distance between the X-ray and dark-matter components and the Mach number of the merger shock.

Results. An approximate solution is given for the problem of the substructure propagation in merging clusters. A new method to predict the position of a dark matter halo in clusters, where there is a separation between the X-ray gas and the dark halo, is proposed and applied to the clusters 1E0657-558 and Abell 1763.
\end{abstract}

Key words. galaxies: clusters: general - galaxies: clusters: individual: 1E0657-558 - galaxies: clusters: individual: Abell 1763 intergalactic medium - shock waves - X-rays: galaxies: clusters

\section{Introduction}

The "unseen" component of the universe (which dominates its mass), is a long-standing issue in modern cosmology since the need for dark matter was originally pointed out by Zwicky (1933). In a typical cluster of galaxies only $2 \%$ of the total mass is in the form of galaxies, $10-15 \%$ is in the form of hot $\mathrm{X}$-ray emitting gas and the remainder is dark matter. Modified Newtonian dynamics (MOND) is an alternative theory that can be used to model different observations without assuming the existence of dark matter (Milgrom 1983). However, MOND cannot account for all the properties of clusters, where the presence of dark matter remains unavoidable (Gerbal et al. 1992; Pointecouteau \& Silk 2006).

The existence of dark matter can be confirmed by the discovery of a system in which the observed baryons and the inferred dark matter are spatially segregated. One type of object where this separation occurs in is merging galaxy clusters. During the collision of two clusters, the galaxies are effectively collisionless particles, while the plasma clouds are highly collisional and therefore are slowed by ram pressure. If dark matter particles are also collisionless, as is widely assumed, any dark matter present in the system would be located near the galaxies. One such merging cluster system is $1 \mathrm{E} 0657-558(z=0.296)$, the socalled bullet cluster. The cluster 1E0657-558 has two primary galaxy concentrations separated by $0.72 \mathrm{Mpc}$ on the sky, a less massive $(T=6 \mathrm{keV})$ western subcluster and a more massive $(T=14 \mathrm{keV})$ eastern main cluster (Markevitch et al. 2002). Both concentrations have associated X-ray emitting plasma offset from the galaxies toward the center of the system (Clowe et al. 2006).

Shock waves driven in the intergalactic medium during the merging of galaxy clusters have been observed in X-ray imaging and spectroscopy. Chandra observations of 1E0657-558 have revealed a bow shock propagating in front of a bullet-like gas cloud moving away from the core of the main cluster. Based on the gas density jump across the shock front, Markevitch et al. (2002) derived a Mach number of the shock 3. The inferred shock velocity of $\sim 4700 \mathrm{~km} \mathrm{~s}^{-1}$ has been commonly interpreted as the velocity of the "bullet" subcluster itself (Markevitch et al. 2002; Hayashi \& White 2006; Markevitch 2006, among others).

Numerical simulations have very recently been run by several teams and succeed quite well in reproducing the main properties of the bullet cluster, both qualitatively and quantitatively. For example, Milosavljevic et al. (2007) conclude that the halo collision velocity need not be the same as the intergalactic gas shock velocity; their simulation finds that the velocity of the CDM halo is $\sim 16 \%$ lower than that of the shocks. Springel \& Farrar (2007) derived a shock speed of about $4500 \mathrm{~km} \mathrm{~s}^{-1}$ but a subcluster velocity of only $2600 \mathrm{~km} \mathrm{~s}^{-1}$ in the rest frame of the system, so the shock wave propagates faster than the dark matter clump. Randall et al. (2007) combine numerical simulations with results derived from X-ray, strong and weak lensing, and optical observations to place an upper limit on the self-interaction crosssection of dark matter per unit mass $\sigma / m$, which rules out most of the range previously invoked to explain inconsistencies between the standard collisionless cold dark matter model and observations. Bradac et al. (2006) have reconstructed the mass distribution of this cluster both from weak and strong lensing data, based on multi band high resolution HST ACS images. They confirmed that the total mass in this cluster does not trace the baryonic mass, as already found by Clowe et al. (2004, 2006).

For the description of the X-ray substructure propagation, we are going to use the "piston in a tube" model, described in Sect. 2. In accordance with this model, an additional retarding force acts on the X-ray substructure. The analysis of substructure propagation is considered in Sect. 3 for various cluster parameters. 
Several estimates from the X-ray observations indicate that around $30 \%$ of all rich clusters exhibit substructure on a scale of $1 \mathrm{Mpc}$ (Forman \& Jones 1994). The number of rich clusters with substructure is a measure of the fraction of galaxy clusters that have recently accreted a significant fraction of their mass (Richstone et al. 1992), which can be used to determine $\Omega_{\mathrm{M}}$.

The dynamical time of the substructure is derived from the approach of Gunn \& Gott (1972) in Sect. 4. Unless evaluations of the incidence of X-ray substructures are overestimated or substructure lasts significantly longer than a gravitational free-fall time in clusters, the value of the fractional density $\Omega_{\mathrm{M}}$ is larger than 0.5 . The retarding force has an effect on the X-ray substructure motion and the duration of the merger can be longer than a gravitational free-fall time because of this force.

We also apply the "piston in a tube" model to the cluster Abell 1763, where the X-ray gas substructure does not seem to have gone beyond the main cluster center. A new method to predict the position of the dark matter halo in clusters, where the observed baryons and the inferred dark matter are spatially segregated, is proposed.

\section{The "piston in a tube" model}

The discovery of a galaxy cluster in which the observed X-ray plasma and the inferred dark matter are spatially segregated requires the existence of a retarding force acting on the X-ray substructure. The observed shock front (in 1E0657-558) moving ahead of the X-ray clump shows supersonic motion of the gas.

We develop a simple 1-D "piston in a tube" model to derive the analytical expression for the retarding force. The retarding force acting on the X-ray clump (piston) in this model arises because the pressure of the compressed gas ahead of the piston is higher than the pressure of the rarefied gas behind the piston. Different regions of the flow are essential for this model: 1) the region of calm gas ahead of the piston; 2) the region of shocked gas; 3) the region of rarefied gas; and 4) the region of calm gas behind the rarefied gas. The piston is situated at the boundary of regions 2, 3 and moves towards region 1 . The shock is situated at the boundary of regions 1,2 .

In regions of calm gas, 1 and 4 , the velocity of the gas equals 0 , the pressure is that of the ambient medium. In region 2 (post-shock region), the velocity of the gas equals the piston velocity $\mathrm{U}$, the pressure is higher than the initial one. In region 3 , the rarefaction wave arises; the velocity of the gas depends on the distance from the piston and the value of the pressure is smaller than the initial one. Qualitative sketches of the gas velocity and pressure profiles in different regions are plotted in Figs. 1 and 2, respectively.

\subsection{The pressure in front of the substructure (region 2)}

Following Landau \& Lifshitz (1959) we calculate the pressure in front of the substructure (region 2). The shock wave arises in front of the substructure (which acts as a piston) because the substructure has a velocity higher than the sound velocity in the main cluster. At first the position of the shock coincides with the position of the piston. Later the shock wave outdistances the piston and a region of shocked gas appears between the shock and the substructure. The gas pressure in front of the shock wave (region 1 ) equals the initial pressure $p_{1}$ and its velocity equals zero. The gas in the region between the shock and the substructure (region 2) has the substructure velocity. The difference between

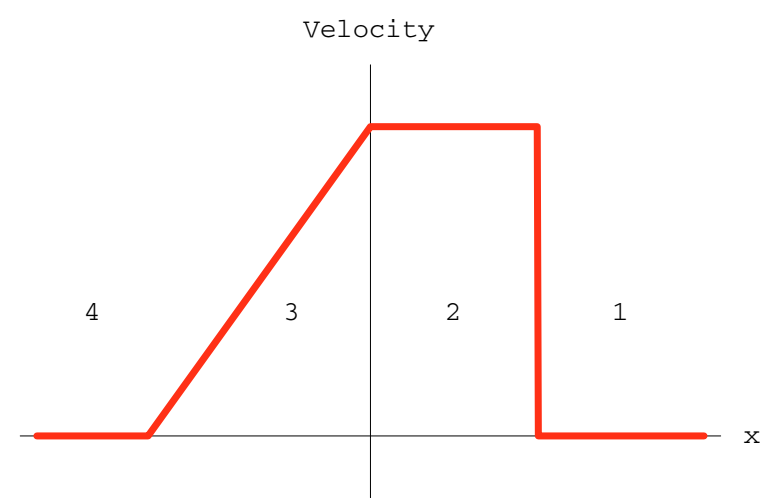

Fig. 1. Gas velocity in different regions of the flow. The piston moves from left to right.

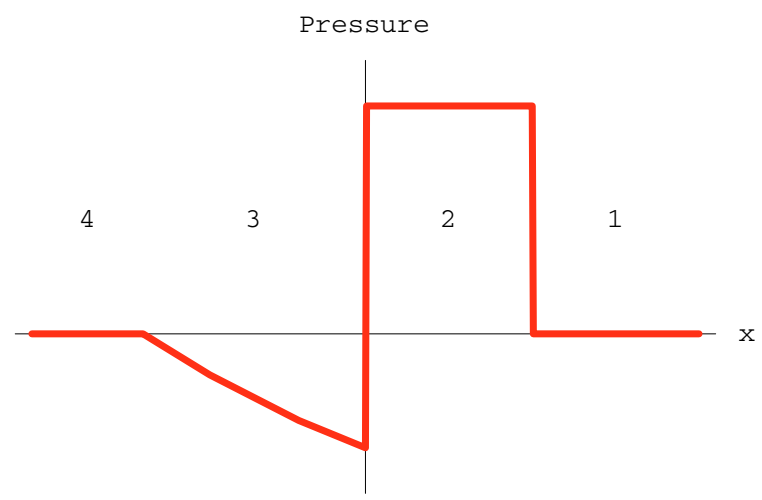

Fig. 2. Gas pressure in different regions of the flow.

the velocities of regions 1 and 2 equals the substructure velocity $U$.

The conditions on the shock (continuity of matter flux, momentum flux, energy flux) can be written as:

$\rho_{1} v_{1}=\rho_{2} v_{2} \equiv j$

$p_{1}+\rho_{1} v_{1}^{2}=p_{2}+\rho_{2} v_{2}^{2}$

$\frac{v_{1}^{2}}{2}+w_{1}=\frac{v_{2}^{2}}{2}+w_{2}$

where $v$ is the gas velocity in the reference frame of the shock, $\rho$ is the gas density, $p$ is the pressure, and $w$ is the specific enthalpy.

From the first equation we find the difference of the velocities:

$v_{1}-v_{2}=\frac{j}{\rho_{1}}-\frac{j}{\rho_{2}}=j \cdot\left(V_{1}-V_{2}\right)$

where $V$ is the inverse of the gas density.

Therefore we can express the matter flux

$j=\frac{v_{1}-v_{2}}{V_{1}-V_{2}}$.

Using the expression $v_{1,2}=j \cdot V_{1,2}$ and Eq. (2), we can find

$p_{1}+j^{2} V_{1}=p_{2}+j^{2} V_{2}$

One can express $j^{2}$ :

$j^{2}=\frac{p_{2}-p_{1}}{V_{1}-V_{2}}$

We can use the Eqs. (5) and (7) together to calculate the velocity $U$ of the substructure:

$U \equiv v_{1}-v_{2}=\sqrt{\left(p_{2}-p_{1}\right)\left(V_{1}-V_{2}\right)}$ 
Using the expression $v_{1,2}=j \cdot V_{1,2}$ and Eq. (3), we can find

$w_{1}+\frac{j^{2} V_{1}^{2}}{2}=w_{2}+\frac{j^{2} V_{2}^{2}}{2}$.

Using Eq. (7), we can find

$w_{1}-w_{2}=\frac{1}{2}\left(V_{1}+V_{2}\right)\left(p_{1}-p_{2}\right)$.

The specific enthalpy of polytropic gas is

$w_{1,2}=\frac{\gamma p_{1,2} V_{1,2}}{(\gamma-1)}$

where $\gamma=c_{p} / c_{v}$.

After the simple transformation of Eqs. (10) and (11), one can find the equation for the percussive adiabat

$\frac{V_{2}}{V_{1}}=\frac{(\gamma+1) p_{1}+(\gamma-1) p_{2}}{(\gamma-1) p_{1}+(\gamma+1) p_{2}}$.

Substituting $V_{2}$ from Eq. (12) in Eq. (8) one can simplify the expression for the substructure velocity

$U=\left(p_{2}-p_{1}\right) \sqrt{\frac{2 V_{1}}{(\gamma-1) p_{1}+(\gamma+1) p_{2}}}$.

For a polytropic gas $p=A \rho^{\gamma}$, therefore the sound velocity:

$c^{2}=\frac{\partial p}{\partial \rho}=\gamma A . \rho^{\gamma-1}=\gamma p V$.

One can find the expression for the pressure in the region 2 from Eqs. (13) and (14):

$\frac{p_{2}}{p_{1}}=1+\frac{\gamma(\gamma+1) U^{2}}{4 c_{1}^{2}}+\frac{\gamma U}{c_{1}} \sqrt{1+\frac{(\gamma+1)^{2} U^{2}}{16 c_{1}^{2}}}$

where $c_{1}$ is the sound velocity in the first region.

\subsection{The pressure behind the substructure (region 3)}

The rarefaction wave is formed behind the substructure (region 3). The self-similarity solution of the flow is described in Landau \& Lifshitz (1959), this solution depends on the single parameter $\xi=x / t$, where $x$ is a distance, $t$ is a time parameter. The equation of entropy conservation is

$\frac{\partial s}{\partial t}+v \frac{\partial s}{\partial x}=0$

where $s$ is the specific entropy, $v$ is the flow velocity.

The derivatives can be expressed as

$$
\begin{aligned}
& \frac{\partial}{\partial t}=-\frac{\xi}{t} \frac{\mathrm{d}}{\mathrm{d} \xi} \\
& \frac{\partial}{\partial x}=\frac{1}{t} \frac{\mathrm{d}}{\mathrm{d} \xi} .
\end{aligned}
$$

Using Eqs. (16) , (17), (18) one can find

$(v-\xi) s_{\xi}^{\prime}=0$

where $s_{\xi}^{\prime} \equiv \mathrm{d} s / \mathrm{d} \xi$.
The Euler equation and the continuity of matter flux equation are

$\frac{\partial v}{\partial t}+v \frac{\partial v}{\partial x}=-\frac{1}{\rho} \frac{\partial p}{\partial x}$
$\frac{\partial \rho}{\partial t}+\rho \frac{\partial v}{\partial x}+v \frac{\partial \rho}{\partial x}=0$.

We can rewrite these equations:

$(v-\xi) \rho_{\xi}^{\prime}+\rho v_{\xi}^{\prime}=0$

$(v-\xi) v_{\xi}^{\prime}=-\frac{c^{2}}{\rho} \rho_{\xi}^{\prime}$.

Consequently from Eqs. (19) and (22), we have $s_{\xi}^{\prime}=0$ and $s=$ const. and the self-similarity solution is isoentropic. Eliminating $\rho_{\xi}^{\prime}, v_{\xi}^{\prime}$ from these two equations, one can find the condition for the non-trivial solution (Landau \& Lifshitz 1959):

$\xi-v=c$

Using the Eqs. (22) and (24), we have

$c \rho_{\xi}^{\prime}=\rho v_{\xi}^{\prime}$

which can be integrated to give $v$ :

$v=\int \frac{c(\rho) \mathrm{d} \rho}{\rho}$.

For adiabatic processes

$\rho T^{1 /(1-\gamma)}=$ const.

The sound velocity is proportional to the square root of the temperature, therefore

$\rho=\rho_{0}\left(\frac{c}{c_{1}}\right)^{2 /(\gamma-1)}$.

Using Eqs. (26) and (28) we have

$v=\frac{2}{\gamma-1} \int \mathrm{d} c=\frac{2}{\gamma-1}\left(c-c_{1}\right)$

where $c_{1}$ is the sound velocity in the region of the calm gas.

$c=c_{1}-\frac{\gamma-1}{2}|v|$.

Using Eq. (28), we obtain the density in the region 3:

$\rho_{3}=\rho_{1}\left(1-\frac{\gamma-1}{2} \frac{|v|}{c_{1}}\right)^{2 /(\gamma-1)}$

and the pressure $p_{3}=A \rho_{3}^{\gamma}$ :

$p_{3}=p_{1}\left(1-\frac{\gamma-1}{2} \frac{|v|}{c_{1}}\right)^{2 \gamma /(\gamma-1)}$. 


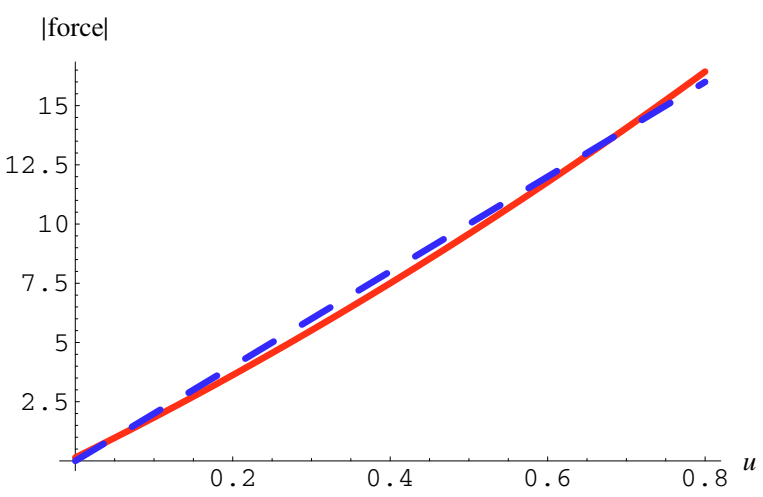

Fig. 3. Functions $F /\left(p_{1} S\right)$ vs. $u$ (solid line) and $G /\left(p_{1} S\right)$ vs. $u$ (dashed line).

\subsection{The retarding force}

From Eqs. (15) and (32) the retarding force $F=\left(p_{3}-p_{2}\right) S$ is:

$$
\begin{aligned}
F= & p_{1} S\left(-\left(1+\frac{\gamma(\gamma+1) U^{2}}{4 c_{1}^{2}}+\frac{\gamma U}{c_{1}} \sqrt{1+\frac{(\gamma+1)^{2} U^{2}}{16 c_{1}^{2}}}\right)\right. \\
& \left.+\left(1-\frac{\gamma-1}{2} \frac{U}{c_{1}}\right)^{\frac{2 \gamma}{\gamma-1}}\right) .
\end{aligned}
$$

Let $\gamma=5 / 3$ and $u=U / 3 c_{1}$, we can rewrite Eq. (33) using these definitions

$F(u)=p_{1} S\left((1-u)^{5}-1-10 u-5 u \sqrt{1+4 u^{2}}\right)$.

If $u \ll 1$, one can calculate the Taylor series

$G(u)=-20 p_{1} S u$.

The functions $F /\left(p_{1} S\right)$ and $G /\left(p_{1} S\right)$ are shown in Fig. 3.

Because of the coincidence of these functions, the function $G(u)$ will be useful later. Thus, we can consider that the retarding function is proportional to the substructure velocity.

\section{Simulation of substructure propagation}

\subsection{Merger initial velocity}

During the merger of two clusters of galaxies, the baryonic component, feeling the gravitational potential created by the dark matter components, moves supersonically (Gabici \& Blasi 2003). Let us assume that two clusters are completely virialized structures with masses $M_{1}$ and $M_{2}\left(M_{2} \ll M_{1}\right.$, e.g., the cluster 1E0657-558). To approximate the merger velocity of the system, we use the assumption (Berrington \& Dermer 2003) that the cluster of mass $M_{2}$ is a point mass. The main cluster accretes another cluster that falls from a turnaround radius $R_{\mathrm{ta}}$ of the system, where the two subclusters are supposed to have zero relative velocity. The exact value of the turnaround radius does not strongly affect the collision velocity as long as it is large and the infall velocity approaches free-fall from infinity. Using the energy conservation law, one can find the velocity of the subcluster with mass $M_{2}$ at the virial radius $R_{\text {virl }}$ of the massive cluster, which has the mass $M_{1}$ :

$$
\begin{aligned}
& \frac{v_{0}^{2}}{2}=\frac{G M_{1}}{R_{\mathrm{vir} 1}}-\frac{G M_{1}}{R_{\mathrm{ta}}} \\
& v_{0}=\sqrt{\frac{2 G M_{1}}{R_{\mathrm{vir} 1}}-\frac{2 G M_{1}}{R_{\mathrm{ta}}}} .
\end{aligned}
$$

The definition of the virial radius is

$$
R_{\mathrm{vir} 1}=\left(\frac{3 M_{1}}{4 \pi \Delta_{\mathrm{c}} \Omega_{\mathrm{M}} \rho_{\mathrm{cr}}}\right)^{1 / 3}
$$

where $\Delta_{\mathrm{c}}$ is the density contrast for the formation of the cluster, $\Omega_{\mathrm{M}}$ is the matter density fraction and the critical density is

$\rho_{\mathrm{cr}}=\frac{3 H^{2}}{8 \pi G}$.

\subsection{Solution without taking the tidal force into account}

The gas number density profiles of X-ray clusters of galaxies can be approximated by the empirical isothermal $\beta$ model (Cavaliere \& Fusco-Femiano 1976)

$n(r)=n_{0}\left(1+\left(\frac{r}{r_{\mathrm{c}}}\right)^{2}\right)^{-3 \beta / 2}$

where $n_{0}$ is the central gas number density, $r_{\mathrm{c}}$ is the core radius of the cluster and $\beta$ is the beta parameter.

Recent high-resolution $N$-body simulations have suggested that dark halos of clusters are described by a family of universal density profiles. Navarro et al. (1997) proposed the following profile (NFW profile):

$\rho_{\mathrm{DM}}(r)=\frac{\delta_{\mathrm{c}} \rho_{\mathrm{cr}} \Omega_{\mathrm{M}}}{\left(r / R_{\mathrm{S}}\right)\left(1+r / R_{\mathrm{S}}\right)^{2}}$

where the characteristic dimensionless density is

$\delta_{\mathrm{c}}=\frac{200}{3} \frac{c^{3}}{\ln (1+c)-c /(1+c)}$
$R_{\mathrm{s}}=R_{\mathrm{vir}} / c$

where $c$ is a concentration parameter (Navarro et al. 1997).

It is useful to introduce a dimensionless time

$\tau=t \frac{c_{\mathrm{s}}}{R_{\mathrm{vir} 1}}$

The initial conditions $x(0), \tilde{v}(0)$ are

$x(0)=-R_{\text {vir } 1}$

$\tilde{v}(0)=\frac{\mathrm{d} x}{\mathrm{~d} \tau}(0)=v_{0} \frac{R_{\mathrm{vir} 1}}{c_{\mathrm{S}}}$

where $\tilde{v} \equiv \mathrm{d} x / \mathrm{d} \tau$.

The gravitational force acting on the X-ray gas of the subcluster equals

$$
\begin{aligned}
F_{\mathrm{gr}}(R)= & \frac{G M_{\mathrm{gas} 2}}{R^{2}} \int_{0}^{R} 4 \pi x^{2} \rho_{\mathrm{DM}}(x) \mathrm{d} x \\
F_{\mathrm{gr}}(R)= & \frac{4 \pi G M_{\mathrm{gas} 2} \delta_{\mathrm{c}} \rho_{\mathrm{cr}} \Omega_{\mathrm{M}} R_{\mathrm{s}}^{3}}{R^{2}} \\
& \times\left(\ln \left(1+\frac{R}{R_{\mathrm{s}}}\right)-\frac{R / R_{\mathrm{s}}}{1+R / R_{\mathrm{s}}}\right)
\end{aligned}
$$

where $M_{\text {gas } 2}$ is the gas mass of the X-ray subcluster.

The equation of motion of the X-ray gas of the substructure without taking into account the tidal force (only the gravitational Eq. (46) and retarding forces Eq. (35) are taken into account) is then:

$$
\begin{aligned}
\frac{\mathrm{d}^{2} x}{\mathrm{~d} \tau^{2}}+\frac{20}{3} & \frac{n(x) k T S R_{\mathrm{vir} 1}}{M_{\mathrm{gas} 2} c_{\mathrm{s}}^{2}} \frac{\mathrm{d} x}{\mathrm{~d} \tau}+4 \pi G \delta_{\mathrm{c}} \rho_{\mathrm{cr}} \Omega_{\mathrm{M}} R_{\mathrm{s}}^{3} \\
& \times \frac{R_{\mathrm{vir} 1}^{2}}{c_{\mathrm{s}}^{2}}\left(\ln \left(1+\frac{|x|}{R_{\mathrm{s}}}\right)-\frac{|x|}{R_{\mathrm{s}}}\left(1+\frac{|x|}{R_{\mathrm{s}}}\right)^{-1}\right) \cdot \frac{|x|}{x^{3}}=0 .
\end{aligned}
$$




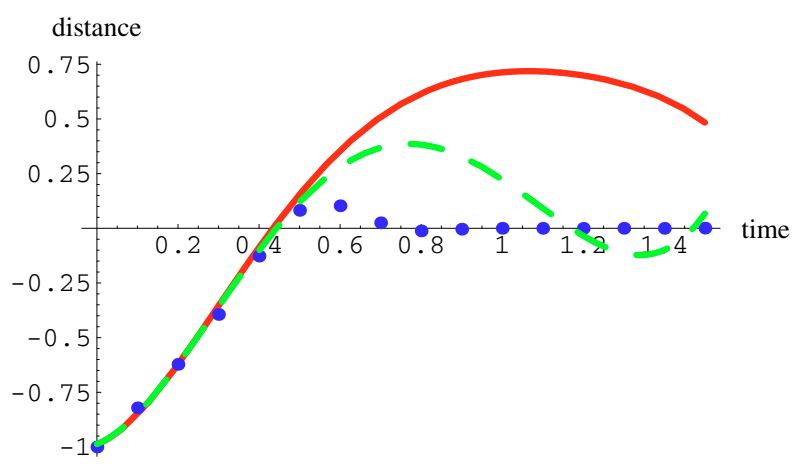

Fig. 4. Dependence of the $\mathrm{X}$-ray substructure position $x / R_{v}$ on the dimensionless time $t R_{v} / c_{\mathrm{s}}$ for different characteristic parameters $\lambda / \omega_{0}=$ 0.25 (solid line), 0.5 (dashed line), 1.0 (dotted line).

The equation of motion of the dark (collisionless) matter substructure is (no retarding force):

$$
\begin{aligned}
\frac{\mathrm{d}^{2} y}{\mathrm{~d} \tau^{2}}=- & 4 \pi G \delta_{\mathrm{c}} \rho_{\mathrm{cr}} \Omega_{\mathrm{M}} R_{\mathrm{s}}^{3} \frac{R_{\mathrm{vir} 1}^{2}|y|}{c_{\mathrm{s}}^{2} y^{3}} \\
& \times\left(\ln \left(1+\frac{|y|}{R_{\mathrm{s}}}\right)-\frac{|y|}{R_{\mathrm{s}}}\left(1+\frac{|y|}{R_{\mathrm{s}}}\right)^{-1}\right) .
\end{aligned}
$$

\subsection{The characteristic parameter of the problem}

The second term on the left-hand side of Eq. (47) weakly depends on the space coordinate in the cluster core. When $x \ll R_{\mathrm{s}}$, the third term on the left-hand side of Eq. (47) can be simplified. One can derive the Taylor series

$\ln \left(1+\frac{|x|}{R_{\mathrm{S}}}\right)-\frac{|x|}{R_{\mathrm{S}}} \cdot\left(1+\frac{|x|}{R_{\mathrm{S}}}\right)^{-1} \sim \frac{x^{2}}{2 R_{\mathrm{S}}^{2}}+o\left(\frac{x^{2}}{R_{\mathrm{S}}^{2}}\right)$.

The simplified expression of Eq. (47) is

$x^{\prime \prime}+\frac{20 n_{0} k T S R_{\mathrm{vir} 1}}{3 M_{\mathrm{gas} 2} c_{\mathrm{s}}^{2}} x^{\prime}+\frac{4 \pi G R_{\mathrm{vir} 1}^{2} \delta_{\mathrm{c}} \rho_{\mathrm{cr}} \Omega_{\mathrm{M}}}{2 c_{\mathrm{s}}^{2}} x=0$.

This equation is the same as the harmonic oscillator one

$x^{\prime \prime}+2 \lambda x^{\prime}+\omega_{0}^{2} x=0$.

The characteristic parameter of such an oscillator motion is

$\frac{\lambda}{\omega_{0}}=\frac{10 n_{0} k T S}{3 M_{\mathrm{gas} 2} c_{\mathrm{s}}} \sqrt{\frac{1}{2 \pi G \delta_{\mathrm{c}} \rho_{\mathrm{cr}} \Omega_{\mathrm{M}}}}$.

The dependence of the X-ray substructure motion with time, which is obtained from Eq. (47) is shown in Fig. 4 for the different values of the characteristic parameter $\left(\lambda / \omega_{0}=0.25,0.5,1\right)$.

The value of $\lambda / \omega_{0}=0.5$ corresponds to the first set of parameters, i.e., to 1E0657-56 (see Table 1).

The characteristic parameter is useful for a classification of the X-ray substructure motion by analogy with an oscillator. If $\lambda / \omega_{0}<1$ the oscillator motion is a dying oscillation, while if $\lambda / \omega_{0}>1$ the oscillator motion is an aperiodic damping.

\subsection{Solution taking the tidal force into account}

It is necessary to consider the influence of the substructure dark matter halo motion on the X-ray substructure motion. The assumption that the small cluster (the dark matter halo and the
Table 1. Sets of parameters for 1E0657-56 and Abell 1763.

\begin{tabular}{lll}
\hline \hline & 1E0657-56 & Abell 1763 \\
Cluster mass $M_{1}, 10^{15} M_{\odot}$ & 2 & 0.5 \\
Central density $n_{0},\left(\mathrm{~cm}^{-3}\right)$ & 0.005 & 0.0079 \\
Core radius $r_{\mathrm{c}},(\mathrm{kpc})$ & 180 & 136 \\
Mass ratio $M_{1} / M_{2}$ & 60 & 22 \\
Subcluster density, $\left(\mathrm{cm}^{-3}\right)$ & 0.02 & 0.0079 \\
Time unit $R_{\mathrm{vir} 1} / c_{\mathrm{s}}, 10^{9} \mathrm{yr}$ & 3.4 & 2.8 \\
$\lambda / \omega_{0}$ & 0.5 & 3.1 \\
\hline
\end{tabular}

Where the NFW concentration parameter is 4 in both cases.

gas substructure) is a point mass is not acceptable because of the infinite gravitational energy at the initial time. It is a reasonable approximation to calculate the tidal force by assuming a NFW profile for the dark matter substructure halo. The tidal force acting on the subcluster dark halo is much smaller than the gravitational force between the two dark halos, because the tidal force is proportional to the mass of the X-ray gas of the subcluster. Consequently the dark matter substructure motion $y(\tau)$ is described by Eq. (48). However, we must include the tidal force in Eq. (47) for the motion of the X-ray substructure by analogy with Eq. (46).

$$
\begin{aligned}
F_{\text {tidal }}=4 & \pi M_{\mathrm{gas} 2} \frac{R_{\mathrm{vir} 1}^{2}}{c_{\mathrm{s}}^{2}} \delta_{\mathrm{c}} \rho_{\mathrm{cr}} \Omega_{\mathrm{M}} R_{\mathrm{s} 2}^{3} \frac{|z-y|}{(y-z)^{3}} \\
& \times\left(\ln \left(1+\frac{|z-y|}{R_{\mathrm{s} 2}}\right)-\frac{|z-y|}{R_{\mathrm{s} 2}}\left(1+\frac{|z-y|}{R_{\mathrm{s} 2}}\right)^{-1}\right)
\end{aligned}
$$

where $z$ is the position of the X-ray subcluster relative to the main cluster center in the case where the tidal force is taken into account.

\subsection{Mach number}

One task is to find the Mach number in the pre-shock region. This is used to determine the temperature ratio of the post-shock region to that in the pre-shock region. One can obtain the dependence of the Mach number in the pre-shock region on the Mach number of the piston:

$\frac{v_{2}}{v_{1}}=\frac{v_{\mathrm{sh}}-U}{v_{\mathrm{sh}}}=\frac{(\gamma-1) M_{1}^{2}+2}{(\gamma+1) M_{1}^{2}}$

where $v_{2}, v_{1}$ are velocities of the post-shock and pre-shock regions. Consequently, in the frame reference of the shock, $v_{\mathrm{sh}}=$ $c_{1} M_{1}$ is the velocity of the shock, $U$ is the velocity of the piston. With $\gamma=5 / 3$, one can find from Eq. (54):

$\frac{U}{c_{1}}=\frac{3}{4} \frac{M_{1}^{2}-1}{M_{1}}$

Therefore the Mach number of the piston is smaller than the Mach number in the pre-shock region.

\subsection{Examples: 1E0657-56 and Abell 1763}

The two sets of selected cluster parameters are in Table 1:

1) for the first type of mergers when the substructure has passed through the main cluster core, the data on the galaxy cluster 1E0657-56 (the "bullet" cluster) are used (Markevitch, private communication); 


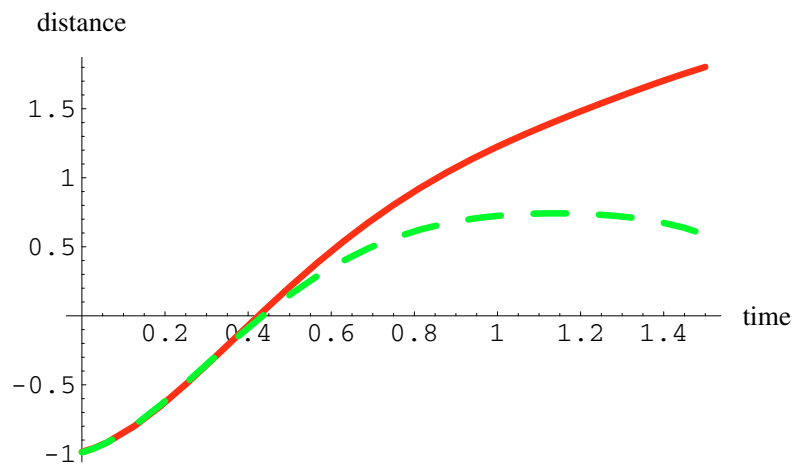

Fig. 5. Dependence of the X-ray substructure position $z / R_{\text {vir1 }}$ (dashed line) and of the dark halo position $y / R_{\mathrm{vir} 1}$ (solid line) on the dimensionless time $t R_{\mathrm{vir} 1} / c_{\mathrm{s}}$ for the first set of parameters (1E0657-56).

2) for the second type of mergers when the substructure has not gone beyond the main cluster center, as in the cluster of galaxies Abell 1763 (Lima Neto \& Durret 2007).

\subsection{E0657-56}

The results can be compared with those of Clowe et al. (2006). The cluster exhibits a prominent bow shock with a Mach number $M=3.0 \pm 0.4$. The distance observed between the two centers, corresponding to the X-ray and dark-matter components of the smaller subcluster, is around $200 \mathrm{kpc}$, and the distance between the centers of the dark-matter substructures is around $1 \mathrm{Mpc}$.

We approximate the shape of the X-ray subcluster with a cylinder (radius $R=79 \mathrm{kpc}$ and length $L=120 \mathrm{kpc}$ ). For the first set of parameters, the position of the X-ray substructure is derived from generalized Eq. (47) taking the tidal force given in Eq. (53) into account, and the position of the dark halo is calculated from Eq. (48); these quantities are plotted in Fig. 5.

The dimensionless time $t R_{v} / c_{\mathrm{s}}=0.51$ corresponds to the observed distance of $1 \mathrm{Mpc}$ between the centers of the two darkmatter substructures and is used to determine the Mach number of the shock and the distance between the X-ray and dark-matter components.

The Mach number of the X-ray substructure $\mathrm{d} z /\left(c_{\mathrm{s}} \mathrm{d} t\right)$ is shown in Fig. 6 and the distance between the X-ray and darkmatter components $y(\tau)-z(\tau)$ is shown in Fig. 7, as functions of the dimensionless time $t R_{v} / c_{\mathrm{s}}$.

From the condition $t R_{v} / c_{\mathrm{s}}=0.51$, we find the value of the Mach number of the X-ray substructure to be $U / c_{1}=2$ from Fig. 6 and the distance between the X-ray and dark-matter components to be $200 \mathrm{kpc}$ from Fig. 7. The Mach number of the shock $M_{1}=3$ is found by solving the quadratic Eq. (55).

Numerical hydrodynamical simulations of the bullet cluster 1E0657-558 (Springel \& Farrar 2007; Milosavljevic et al. 2007) reached a very similar conclusion to ours: the halo collision velocity need not be the same as the intergalactic gas shock velocity, and while the kinematics of the shock are sensitive to the details of the cluster structure, the instantaneous shock velocity can exceed the relative velocity of CDM halos. 2D numerical simulations provide much more precise results than our approximate theory, because we made several simplifying assumptions (e.g., the problem is 1-D), and for accurate description of merging clusters numerical simulations are very useful. On the other hand, numerical simulations provide information for very specific sets of parameters. One immediate question is what are the key controlling parameters and might they affect

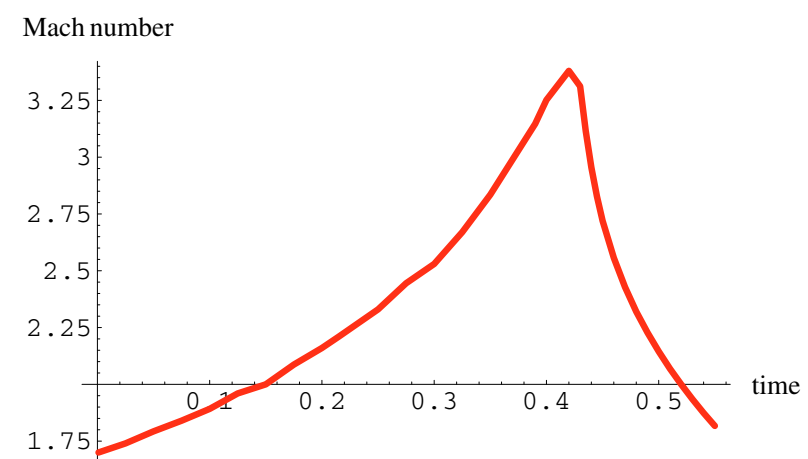

Fig. 6. Mach number of the X-ray structure vs the dimensionless time $t R_{\mathrm{vir} 1} / c_{\mathrm{s}}$ for $1 \mathrm{E} 0657-56$.

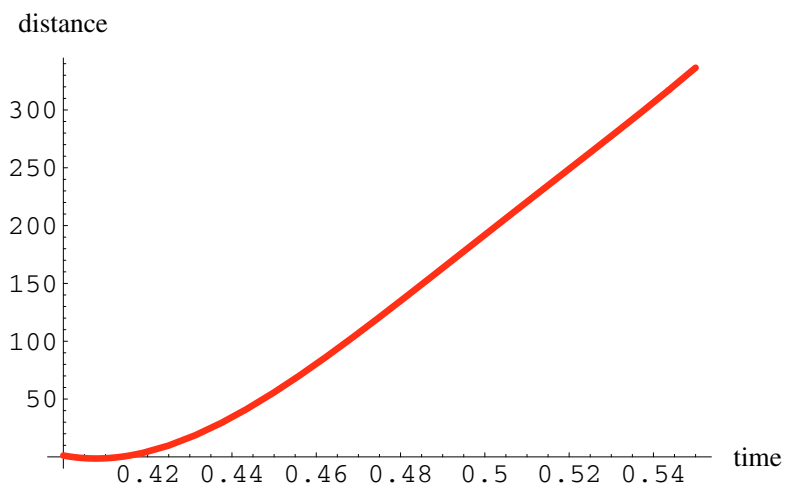

Fig. 7. Dependence of the distance $(\mathrm{kpc})$ between the X-ray and darkmatter substructures on the dimensionless time $t R_{\mathrm{virl}} / c_{\mathrm{s}}$ for 1E0657-56.

the results? For example, using different simulation parameters Milosavljevic et al. (2007) and Springel \& Farrar (2007) find that the velocities of the CDM halos are respectively $\sim 16 \%$ and $\sim 40 \%$ lower than that of the shock. Milosavljevic et al. (2007) strongly emphasize that the shock kinematics and morphology are extraordinarily sensitive to the parameters of the simulations. The advantage of our approximative theory is that it has a single characteristic parameter $\lambda / \omega_{0}$, which completely determines the $\mathrm{X}$-ray substructure motion.

The similar results of Milosavljevic et al. (2007) and Springel \& Farrar (2007), i.e., that the shock velocity is higher than the dark halo velocity, are expected for merging clusters, where the characteristic parameter $\lambda / \omega_{0}$ is much smaller than 1 . The influence of the retarding force in these systems is negligible and the velocity of the shock is higher than those of the X-ray substructure and the dark halo, because the shock outdistances the piston in the "piston in a tube" model.

\subsection{Abell 1763}

We assume that the X-ray subcluster has the shape of a sphere and the radius of the X-ray subcluster equals the radius of the hottest region in the cluster, that is $R \approx 140 \mathrm{kpc}$. We propose that the hottest region in Abell 1763 (where the temperature is twice the average temperature) is situated in the post-shock region. From the Rankine-Hugoniot condition we can estimate the Mach number in the pre-shock region. The ratio of the temperatures in the two regions is:

$\frac{T_{2}}{T_{1}}=\frac{\left(2 \gamma M_{1}^{2}-(\gamma-1)\right)\left((\gamma-1) M_{1}^{2}+2\right)}{(\gamma+1)^{2} M_{1}^{2}}$ 


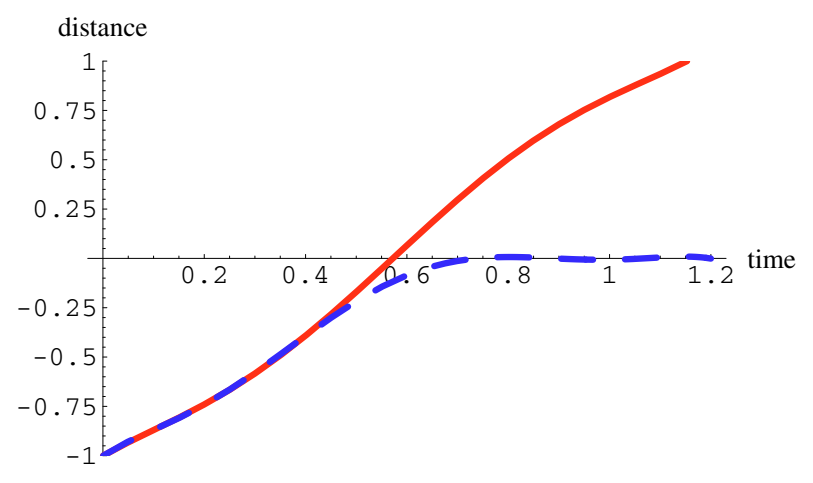

Fig. 8. Dependence of the $\mathrm{X}$-ray substructure position $z / R_{\text {vir1 }}$ (dashed line) and of the dark halo position $y / R_{\mathrm{vir} 1}$ (solid line) on the dimensionless time $t R_{\mathrm{virl}} / c_{\mathrm{s}}$ for the second set of parameters (Abell 1763).

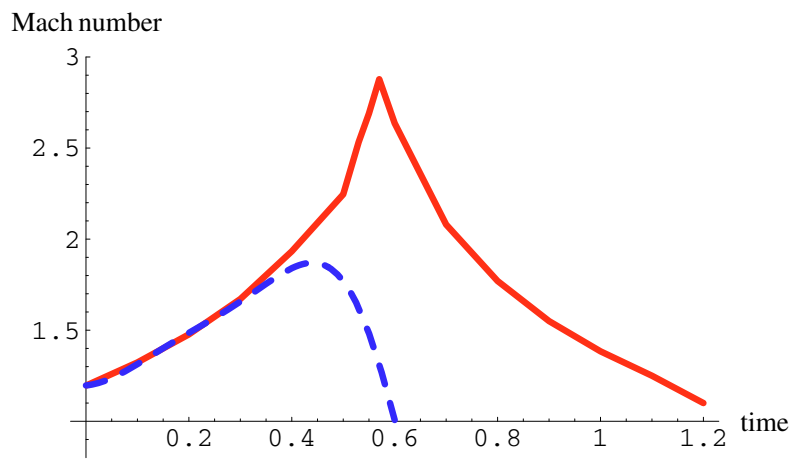

Fig. 9. Mach number of the piston (dotted line) and of the dark halo (solid line) vs. dimensionless time $t R_{\mathrm{vir}} / c_{\mathrm{s}}$ for Abell 1763.

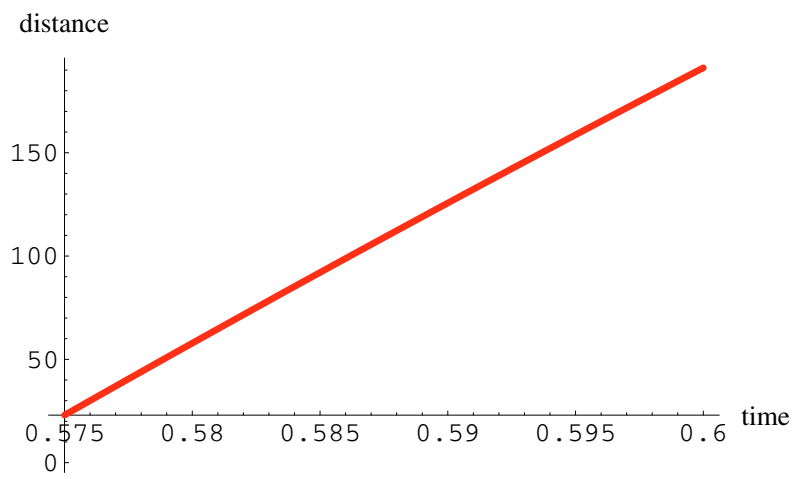

Fig. 10. Position of the dark matter substructure halo $(\mathrm{kpc})$ vs. dimensionless time $t R_{\mathrm{virl}} / c_{\mathrm{s}}$ for Abell 1763.

Using Eq. (55) we find the Mach number of the piston to be $U / c_{1}=1.1$. One can see from Fig. 9 that the dark matter substructure has a much higher velocity than the X-ray substructure. Therefore the effect of the retarding force is stronger in this cluster than in 1E0657-56. One can therefore expect a separation of the dark matter halo of the substructure and the X-ray substructure (Fig. 10). If the galaxies and X-ray subclump are not segregated, a galaxy concentration near the region of the substructure dark matter halo is expected about $200 \mathrm{kpc}($ time $=0.6)$ from the center of the cluster Abell 1763 in the direction of the merger (south east to north west).

In order to check this point, we analyzed an optical image of Abell 1763 in the $R$ band taken with the LFC camera on the Palomar telescope and kindly provided by Fadda and Biviano.
The SExtractor software was applied to the image, and stars were discarded on the basis of a plot of effective radius as a function of magnitude, leading to a catalogue of galaxies. From a magnitude histogram, the galaxy catalogue was estimated to be complete up to $R \sim 24$. We therefore truncated the catalogue at this magnitude to avoid effects due to incompleteness.

Galaxies from this catalogue were then counted in squares of $140 \times 140 \mathrm{kpc}^{2}$ centered on the cluster center. The numbers of galaxies in each square were found to be between 24 and 52, with the lowest number in the NW region, where our model predicts an overabundance of galaxies. The contamination by background galaxies is estimated to be 9 galaxies in each square, as derived from the AAO model by Bland-Hawthorn \& Ellis (http://www.aao.gov.au/astro/GalaxyCount/).

There is therefore no galaxy excess $200 \mathrm{kpc} N W$ of the cluster center, indicating that the X-ray gas of the subcluster must have stopped while the galaxies have had time to travel further out. Hence, a direct correspondence between the hottest region in Abell 1763 and the post-shock region is not correct. Abell 1763 is therefore a structure in which the observed baryons of the subcluster and the galaxy concentration are spatially segregated; a full analysis of the optical image will be done elsewhere and is beyond the scope of this paper.

Dark matter annihilation in the halos of galaxy clusters has relevant astrophysical implications (e.g., Colafrancesco 2006). In fact, if dark matter is constituted by weakly interacting massive particles (WIMPs), their annihilation can produce direct observable signals. However, the spatial and spectral intensities of the astrophysical signals coming from $\chi \chi$ annihilation are expected to be confused with, or even overcome by, other astrophysical signals originating from the intracluster gas and/or from the relativistic plasmas present in the cluster atmospheres, especially when all these components are co-spatially distributed with the DM component. An ideal system to detect a DM annihilation signal would therefore be a cluster with a clear spatial separation between the various matter components (Colafrancesco et al. 2007).

\section{Evaluation of the incidence of substructures}

Clusters of galaxies form by the mergers of smaller clusters and groups. The presence of substructure in clusters of galaxies can be used to estimate the cosmological density parameter $\Omega_{\mathrm{M}}$.

$\mathrm{X}$-ray data allow the quantification of substructures because cluster mergers compress and heat the intracluster gas, and this can be measured as distortions of the spatial distribution of X-ray surface brightness and temperature (Forman \& Jones 1994; Jones \& Forman 1999). An alternative method at optical wavelengths to study substructures is based on the distribution of cluster galaxies. However, the optical method requires a large number of galaxies (Dutta 1995), at least a few hundred, and is more susceptible to contamination from foreground and background objects.

$X$-ray studies of cluster substructure use a number of different statistics (see Buote 2002 for a review). Several studies have used the ellipticity (Gomez et al. 1997; Kolokotronis et al. 2001; Melott et al. 2001; Plionis 2002), while other studies measured centroid (or center-of-mass) variations (Mohr et al. 1995; Gomez et al. 1997; Kolokotronis et al. 2001). Another method developed by Buote \& Tsai (1995) is the power ratio method, which is constructed from the moments of the X-ray surface brightness.

Richstone et al. (1992) performed the first theoretical study of the relationship of substructure to cosmology. They assumed that any substructure is wiped out in a cluster crossing time and 
calculated the fraction of clusters in the spherical growth approximation that formed within the last crossing time as a function of $\Omega_{M}$ and $\Lambda$. This fraction primarily depends on $\Omega_{M}$, and they estimated that $\Omega_{\mathrm{M}}>0.5$ based on estimates of the frequency of substructure in low-redshift clusters from X-ray images.

The approach proposed by Richstone et al. (1992) to determine $\Omega_{\mathrm{M}}$ suffers several limitations. In their semi-analytical calculations they avoided the issue of the power spectrum by concentrating on clusters having the same total mass. The collapse time of a $10^{15} M_{\odot}$ spherical density perturbation was defined to be the dividing point between clusters that do and do not possess substructure. The relationship between the collapse time of a spherical density perturbation and subclustering, though qualitatively reasonable, is ambiguous. Consequently, they predict an ambiguous "frequency of substructure" rather than a welldefined quantitative measure of cluster morphology.

A more recent semi-analytical approach was developed by Buote (1998). He assumed that the amount of substructure depends on the amount of mass accreted by a cluster over a relaxation timescale and relates the fractional accreted mass to the power ratios. Although these semi-analytical methods give an indication of the expected evolution of cluster substructure and its dependence on cosmological parameters, perhaps the best method to constrain cosmological models is through the comparison to cluster simulations.

Evrard et al. (1993) employed $N$-body/3D gas dynamic simulations of the formation of galaxy clusters and demonstrated the existence of a morphology-cosmology connection for X-ray clusters. Confirming the analytical expectations of Richstone et al. (1992), they showed that cluster evolution is sensitive to the cosmological model in which the clusters form. Numerical simulations show that both the centroid shift and the power ratios are capable of distinguishing cosmological models (Evrard et al. 1993; Buote \& Xu 1997; Valdarnini et al. 1999; Suwa et al. 2003). Buote \& Xu (1997) find that the power ratios of their ROSAT clusters indicate an $\Omega_{\mathrm{M}}<1$ universe. Valdarnini et al. (1999) find that the $\Lambda$ CDM model is inconsistent with the data. However, they used $\sigma_{8}=1.1$, which is fairly high and may cause the disagreement between the $\Lambda$ CDM model and the data. Suwa et al. (2003) find that the center shifts and power ratios of both surface brightness and projected mass density are able to discriminate between the cosmological models at $z=0$.

We use the value of $\Omega_{M}=0.268 \pm 0.018$, which was obtained from the WMAP three year observations (Spergel et al. 2007), to find the dynamical time of the substructure, which is higher than the gravitational free-fall time. A plausible reason for this disagreement is that the fraction of clusters with X-ray substructures exceed the number of clusters that have collapsed during the last gravitational free-fall time due to the retarding force acting on the X-ray substructure.

\subsection{The collapse time for bound perturbations}

The separation of two observers in a Friedman-RobertsonWalker universe with a cosmology constant obeys the equation of motion

$\frac{\mathrm{d}^{2} r}{\mathrm{~d} t^{2}}=-\frac{4 \pi G \rho_{\star} r_{\star}^{3}}{3 r^{2}}+\frac{\Lambda r}{3}$

where $\rho_{\star}, r_{\star}$ are the mean density of the universe and the separation between two observers at any specified epoch in the universe.

This equation also describes the evolution of the central distance of a point anywhere in a homogeneous perturbation.
The equation of mass conservation shows that $\Omega_{\mathrm{M}}(z)$ and $\lambda(z)$ of the universe evolve as

$$
\begin{aligned}
& \Omega_{\mathrm{M}}(z)=\frac{(1+z)^{3} \Omega_{\mathrm{M} 0}}{H^{2}(z) / H_{0}^{2}} \\
& \lambda(z)=\frac{\lambda_{0}}{H^{2}(z) / H_{0}^{2}}
\end{aligned}
$$

where $\Omega \equiv 8 \pi G \rho /\left(3 H^{2}\right), \lambda=\Lambda /\left(3 H^{2}\right)$.

The dependence of the rate of expansion $H(z)$ is given by

$$
\frac{H^{2}(z)}{H_{0}^{2}}=\left(1-\Omega_{\mathrm{M} 0}-\lambda_{0}\right)(1+z)^{2}+\Omega_{\mathrm{M} 0}(1+z)^{3}+\lambda_{0} .
$$

It is possible to rewrite Eq. (57) in integral form and estimate the time to expand from $r_{\star}$ to $r$ for a homogeneous perturbation:

$T=H_{\star}^{-1} \int_{1}^{r / r_{\star}}\left(1-\Omega_{\mathrm{M} \star}-\lambda_{\star}+\Omega_{\star} u^{-1}+\lambda_{\star} u^{2}\right)^{-1 / 2} \mathrm{~d} u$,

Eq. (60) is only valid for perturbations that expand at least as far as $r / r_{\star}$. Bound perturbations which do not expand forever reach a maximum expansion and recollapse on a time scale given by

$\tau=2 T\left(r_{\mathrm{m}} / r_{\star}\right)$

where $r_{\mathrm{m}}$ is the first real root $>1$ of the equation:

$\left(1-\Omega_{\mathrm{M} \star}-\lambda_{\star}\right)+\Omega_{\mathrm{M} \star} u^{-1}+\lambda_{\star} u^{2}=0$.

\subsection{Initial density perturbations}

Now suppose that at the epoch defined by $1+z_{1}=1000$ the universe is characterized by an unperturbed Hubble flow and cosmological constant, but that the density, on the mass scales of clusters of galaxies, is normally distributed about the mean density with variance $\sigma^{2}$. Thus, we can write for the perturbations $\delta$ :

$\lambda_{\star}=\lambda\left(z_{1}\right)$

$\Omega_{\mathrm{M} \star}=\Omega_{\mathrm{M}}\left(z_{1}\right) \cdot(1+\delta)$

and $\delta$ has the Gaussian distribution

$\mathrm{d} F(\delta)=\frac{1}{\sqrt{2 \pi \sigma^{2}}} \exp \left(-\frac{\delta^{2}}{2 \sigma^{2}}\right) \mathrm{d} \delta$.

The bound perturbation of the density must exceed the critical value $\delta_{\mathrm{cr}}$, which is given by

$$
\begin{aligned}
1-\Omega_{\mathrm{M}}\left(z_{1}\right) \cdot\left(1+\delta_{\mathrm{cr}}\right)-\lambda\left(z_{1}\right) & \\
& +1.5 \cdot\left[2 \lambda\left(z_{1}\right) \cdot\left(\Omega_{\mathrm{M}}\left(z_{1}\right) \cdot\left(1+\delta_{\mathrm{cr}}\right)\right)^{2}\right]^{1 / 3}=0 .
\end{aligned}
$$

One can calculate from Eq. (65) the critical value of perturbations $\delta_{\text {cr }}=2.5 \times 10^{-3}$.

The maximum expansion factor for a homogeneous spherical perturbation is given by Eq. (62).

Direct substitution in Eqs. (60), (61) and (63) yields the ratio of the collapse time to the current age of the (unperturbed) universe for bound perturbations

$$
\begin{aligned}
& \frac{\tau}{T_{0}}= \\
& \frac{2 \int_{1}^{r_{\mathrm{m}} / r_{1}} \mathrm{~d} u\left(1-\Omega_{\mathrm{M}}(1+\delta)-\lambda+\Omega_{\mathrm{M}}(1+\delta) u^{-1}+\lambda u^{2}\right)^{-1 / 2}}{\int_{1}^{1+z_{1}} \mathrm{~d} u\left(1-\Omega_{\mathrm{M}}-\lambda+\Omega_{\mathrm{M}} u^{-1}+\lambda u^{2}\right)^{-1 / 2}}
\end{aligned}
$$

where $\Omega_{\mathrm{M}}, \lambda$ are fixed at the values evaluated at $1+z_{1}$. 


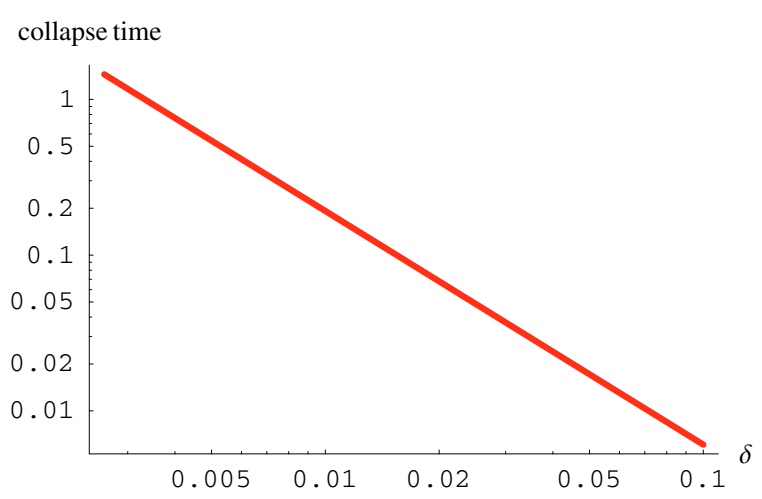

Fig. 11. Collapse time $\tau / T_{0}$ as a function of the density perturbation $\delta$.

\subsection{The estimation of the dynamical time}

For a particular universe, specified by $\Omega_{\mathrm{M} 0}, \lambda_{0}$, Eq. (66) defines a monotonic relation between $\delta$ and $\tau / T_{0}$ for bound perturbations, and can therefore be regarded as an equation for $\delta$ as a function of $\tau / T_{0}$ (Richstone et al. 1992). Equations (64) and (66) specify, for a particular $\Omega_{\mathrm{M} 0}, \lambda_{0}, \sigma$, the fraction of the universe on some specific mass scale that has already collapsed at cosmic time t:

$F\left(t / T_{0}\right)=\frac{1}{2}\left(1-\operatorname{erf}\left(\frac{\delta\left(t / T_{0}\right)}{\sqrt{2} \sigma}\right)\right)$

where erf is the error function.

Equation (67) predicts the fraction of the universe currently in virialized clusters with a mass scale of rich Abell clusters. That fraction is the product of the number density $\langle n\rangle$ and mass of rich clusters $M$, divided by the mean density of the universe. The value of $\sigma$ must be found by solving Eq. (68):

$\frac{1}{2}\left(1-\operatorname{erf}\left(\frac{\delta(1)}{\sqrt{2} \sigma}\right)\right)=\frac{\langle n\rangle M}{\rho_{\mathrm{cr}} \Omega_{\mathrm{M} 0}}$.

The simplification of Eq. (66) is useful from an analytical point of view in order to find the function $\delta\left(\tau / T_{0}\right)$. A good approximation can be found by assuming

$\tau(\delta) \propto \delta^{-3 / 2}$.

The function $\tau / T_{0}$ computed from Eq. (66) is shown in Fig. 11.

The fraction of the mass of present day clusters that have collapsed within the time interval $\left(T_{0}-\delta t, T_{0}\right)$ given in Fig. 12 is

$\delta \tilde{F}\left(\frac{\delta t}{T_{0}}\right)=\frac{F(1)-F\left(1-\delta t / T_{0}\right)}{F(1)}$

Forman \& Jones (1994) have studied the X-ray surface brightness of 250 clusters of galaxies observed with Einstein. They concluded that $30 \%$ of that sample had X-ray substructure. Taking into account this observational constraint one can derive the dynamical time, and we find $\delta t / T_{0}=0.17$ (the big point in Fig. 12).

\subsection{The retarding of an $X$-ray substructure}

Since Eq. (51) can be solved analytically, we estimate the retarding of an X-ray substructure from this equation when the characteristic parameter $\lambda^{2} / \omega_{0}^{2} \gg 1$.

The solution of Eq. (51) in case $\lambda / \omega_{0}>1$ with initial conditions $(x(0)=-R, v(0)=0)$ is

$$
\begin{aligned}
x= & \left(C_{1}+C_{2}\right) \exp \left(-\left(\lambda-\sqrt{\lambda^{2}-\omega^{2}}\right) \cdot t\right) \\
& +\left(C_{1}-C_{2}\right) \exp \left(-\left(\lambda+\sqrt{\lambda^{2}-\omega^{2}}\right) \cdot t\right)
\end{aligned}
$$

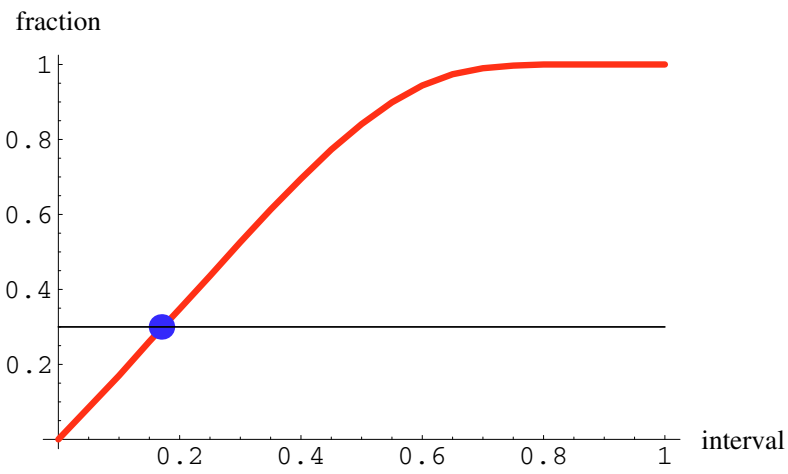

Fig. 12. The fraction of the mass of present day clusters that have collapsed within the last time interval $\delta t / T_{0}$.

where

$$
\begin{aligned}
& C_{1}=-R / 2 \\
& C_{2}=-\frac{\lambda R}{2 \sqrt{\lambda^{2}-\omega_{0}^{2}}} .
\end{aligned}
$$

The X-ray substructure velocity is found from the Eq. (71):

$$
\frac{\mathrm{d} x}{\mathrm{~d} t}=A \mathrm{e}^{-\lambda t} \operatorname{ch}\left(\sqrt{\lambda^{2}-\omega_{0}^{2}} \cdot t\right)+\mathrm{Be}^{-\lambda t} \operatorname{sh}\left(\sqrt{\lambda^{2}-\omega_{0}^{2}} \cdot t\right)
$$

where

$$
\begin{aligned}
& A=2\left(-\lambda C_{1}+\sqrt{\lambda^{2}-\omega_{0}^{2}} C_{2}\right) \\
& B=2\left(-\lambda C_{2}+\sqrt{\lambda^{2}-\omega_{0}^{2}} C_{1}\right) .
\end{aligned}
$$

If the characteristic parameter $\lambda^{2} / \omega_{0}^{2} \gg 1$, we have

$$
\begin{aligned}
& \exp \left(-\lambda t+\sqrt{\lambda^{2}-\omega_{0}^{2}} t\right) \approx \exp \left(-\frac{\omega_{0}^{2}}{2 \lambda} t\right) \\
& \exp \left(-\lambda t-\sqrt{\lambda^{2}-\omega^{2}} t\right) \approx \exp (-2 \lambda t) .
\end{aligned}
$$

Therefore when the time $t \gg 1 / \lambda$, we can consider only the terms of Eq. (73) that contain the factor $\exp \left(-\lambda t+\sqrt{\lambda^{2}-\omega_{0}^{2}} t\right)$. The dependence of the velocity with time $(t \gg 1 / \lambda)$ is

$\frac{\mathrm{d} x}{\mathrm{~d} t} \approx R \frac{\omega_{0}^{2}}{2 \lambda} \exp \left(-\frac{\omega_{0}^{2}}{2 \lambda} t\right)$.

The derived velocity of the X-ray substructure in case $\lambda / \omega_{0}>1$ is much smaller than the dark halo velocity $\left(\sim R \omega_{0}\right)$. Therefore for merger events, when this characteristic parameter is much higher than 1, the dynamical time is much higher than the gravitational free-fall time. This argument can explain that the merger duration is higher than the gravitational free-fall time for many merger events.

\section{Conclusions}

There are many proofs of evidence that X-ray substructures move with a supersonic velocity in the ICM of clusters. In their recent review of shocks and cold fronts in galaxy clusters, Markevitch \& Vikhlinin (2007) nicely summarize our present knowledge on physical mechanisms taking place when clusters merge, such as those discussed in the present paper. The 
analytical study of the X-ray substructure motion is proposed in the present article. The retarding force must be taken into account to obtain a correct solution. One of the most obvious manifestations of its importance is the spatial segregation of the stellar component and the X-ray substructure in the galaxy cluster 1E0657-558. The behavior of the X-ray substructure depends on the characteristic parameter (see Fig. 4) in the "piston in a tube" model. When the characteristic parameter is smaller than 1 , the $\mathrm{X}$-ray substructure can pass through the main cluster core (e.g. 1E0657-558). We also examine the case when the characteristic parameter is higher than 1 and the effect of the retarding force is crucial (e.g. Abell 1763). The shock velocity in front of the $\mathrm{X}$-ray substructure is calculated. The method for predicting the position of the dark matter halo in clusters, where the observed baryons and the inferred dark matter are spatially segregated, is considered.

Richstone et al. (1992) used the dynamical time of the substructure to constrain $\Omega_{\mathrm{M}}$, but their constraint $\Omega_{\mathrm{M}}>0.5$ does not correspond to the current data. A possible reason for this is that substructure lasts significantly longer than a crossingtime in clusters. The retarding force influences the substructure dynamical time, which is then higher than the crossing-time. Consequently, an accurate description of substructure propagation is a requisite to estimate $\Omega_{\mathrm{M}}$.

Acknowledgements. The authors thank the referee for useful comments, and G. B. Lima Neto and M. Markevitch for discussions. They are very grateful to D. Fadda for providing the optical image of Abell 1763 and to M. Montessuit and $\mathrm{G}$. Boué for their help in analyzing this image.

\section{References}

Berrington, R. C., \& Dermer, C. D. 2003, ApJ, 594, 709

Bradac, M., Clowe, D., Gonzales, A. H., et al. 2006, ApJ, 652, 937

Buote, D. A. 1998, MNRAS, 293, 381

Buote, D. A. 2002, in Mergering Processes in Galaxy Cluster, ed. L. Feretti, I. M. Gioia, \& G. Giovannini (Dordrecht: Kluwer), 79 [arXiv:astro-ph/0106057]
Buote, D. A., \& Tsai, J. C. 1995, ApJ, 452, 522

Buote, D. A., \& Tsai, J. C. 1997, MNRAS, 284, 439

Cavaliere, A., \& Fusco-Femiano, R. 1976, A\&A, 49, 137

Clowe, D., Bradac, M., Gonzalez, A. H., et al. 2006, ApJ, 648, L109

Colafrancesco, S. 2006, Chin. J. Astron. Astrophys., 6, 95

Colafrancesco, S., de Bernardis, P., Masi, S., Polenta, G., \& Ullio, P. 2007, A\&A, 467, L1

Dutta, S. N. 1995, MNRAS, 276, 1109

Evrard, A. E., Mohr, J. J., Fabricant, D. G., \& Geller, M. J. 1993, ApJ, 419, L9

Gabici, S., \& Blasi, P. 2003, ApJ, 583, 695

Gerbal, D., Durret, F., Lachièze-Rey, M., \& Lima Neto, G. B. 1992, A\&A, 262, 395

Gomez, P. L., Pinkney, J., Burns, J. O., et al. 1997, ApJ, 474, 580

Gunn, J. E., \& Gott, J. R. 1972, ApJ, 176, 1

Forman, W., \& Jones, C. 1994, in Cosmological Aspects of X-Ray Clusters of Galaxies, ed. W. C. Seitter (Kluwer)

Hayasi, E., \& White, S. D. M. 2006, MNRAS, 351, 423

Jones, C., \& Forman, W. 1999, ApJ, 511, 65

Kolokotronis, V., Basilakos, S., Plionis, M., \& Georgantopoulos, I. 2001, MNRAS, 320, 49

Landau, L. D., \& Lifshitz, E. M. 1959, Fluid Mechanics (Addison-Wesley Reading)

Lima Neto, G. B., \& Durret, F. 2007, A\&A, submitted

Markevitch, M., Gonzalez, A. H., David, L., et al. 2002, ApJ, 567, L27

Markevitch, M. 2006, ESA SP-604: The X-ray Universe 2005, 723

Markevitch, M., \& Vikhlinin, A. 2007, Phys. Rep., 443, 1

Melott, A., Chambers, S. W., \& Miller, C. J. 2001, ApJ, 546, 100

Milgrom, M. 1983, ApJ, 270, 365

Milosavljevic, M., Koda, J., Nagai, D., Nakar, E., \& Shapiro, P. R. 2007, ApJ, 661, L131

Mohr, J. J., Evrard, A. E., Fabricant, D. G., \& Gelle, M. J. 1995, ApJ, 447, 8

Navarro, J. F., Frenk, C. S., \& White, S. D. M. 1997, ApJ, 490, 493

Plionis, M. 2002, ApJ, 572, 67

Pointecouteau, E., \& Silk, J. 2005, MNRAS, 364, 654

Randall, S. W., Markevitch, M., Clowe, D., Gonzales, A. H., \& Bradac, M. 2007 [arXiv: astro-ph/07040261]

Richstone, D., Loeb, A., \& Turner, E. L. 1992, ApJ, 393, 477

Spergel, D. N., Bean, R., \& Doré, O. 2007, ApJS 170, 377

Springel, V., \& Farrar, G. 2007, MNRAS, 380, 911

Suwa, T., Habe, A., Yoshikawa, K., \& Okamoto, T. 2003, ApJ, 588, 7

Valdarnini, R., Ghizzardi, S., \& Bonometto, S. 1999, New Astron., 4, 71

Zwicky, F. 1933, Helv. Phys. Acta, 6, 110 